\title{
Effect of Colchicine on Platelet-Platelet and Platelet-Leukocyte Interactions: A Pilot Study in Healthy Subjects
}

\author{
Binita Shah, MD, MS ${ }^{\star}$, , Nicole Allen, BS ${ }^{\star}$, Bhisham Harchandani, MD*, Michael Pillinger, \\ MD ${ }^{\ddagger}$, Stuart Katz, MD ${ }^{\star}$, Steven P. Sedlis, MD $^{\star}, \dagger$, Christina Echagarruga, MS ${ }^{\star}$, Svetlana \\ Krasnokutsky Samuels, MD, MS $\ddagger$, Pajazit Morina, MD*, Prabhjot Singh, MD, Liza Karotkin, \\ BA $^{*}$, and Jeffrey S. Berger, MD, MS $\S$ \\ *Department of Medicine, Division of Cardiology, New York University School of Medicine, New \\ York, NY \\ †Department of Medicine, Section of Cardiology, Veterans Affairs New York Harbor Health Care \\ System, New York, NY \\ FDepartment of Medicine, Division of Rheumatology, New York University School of Medicine, \\ New York, NY \\ §Department of Medicine, Division of Cardiology and Hematology, New York University School of \\ Medicine, New York, NY
}

\begin{abstract}
The cardioprotective mechanisms of colchicine in patients with stable ischemic heart disease remain uncertain. We tested varying concentrations of colchicine on platelet activity in vitro, and a clinically relevant $1.8 \mathrm{mg}$ oral loading dose administered over one hour in 10 healthy subjects. Data are shown as median [interquartile range]. Colchicine addition in vitro decreased light transmission platelet aggregation only at supratherapeutic concentrations, but decreased monocyte- (MPA) and neutrophil-platelet aggregation (NPA) at therapeutic concentrations. Administration of $1.8 \mathrm{mg}$ colchicine to healthy subjects had no significant effect on light transmission platelet aggregation but decreased the extent of MPA (28\% [22-57] to 22\% [19-31], $\mathrm{p}=0.05)$ and NPA (19\% [16-59] to 15\% [11-30], $\mathrm{p}=0.01)$, platelet surface expression of PAC-1 (370 mean fluorescence intensity (MFI) [328-555] to 333 MFI [232-407], $\mathrm{p}=0.02$ ) and P-selectin (351 MFI [269-492] to 279 [226-364], $\mathrm{p}=0.03)$, and platelet adhesion to collagen (10.2\% [2.532.6 ] to $2.0 \%$ [0.2-9.5], $\mathrm{p}=0.09) 2$ hours post-administration. Thus, in clinically relevant concentrations, colchicine decreases expression of surface markers of platelet activity and inhibits leukocyte-platelet aggregation, but does not inhibit homotypic platelet aggregation.
\end{abstract}

\section{Keywords}

colchicine; platelet aggregation; neutrophil; monocyte; tissue adhesion

Corresponding author: Binita Shah, MD, MS, 227 E $30^{\text {th }}$ Street, Office 835, New York, NY 10016, Telephone: 212-263-4235, Fax: 212-263-8534, binita.shah@nyumc.org. 


\section{Introduction}

Colchicine is an anti-inflammatory agent commonly used in rheumatic diseases and pericarditis [1]. Recent data suggest potential benefit in other systemic inflammatory disease processes, including atherosclerotic heart disease [2-3]. The cardioprotective mechanisms of colchicine have not been fully elucidated. Microtubules support the discoid shape of platelets and may play a role in platelet activation [4-8]. Colchicine disrupts microtubule polymerization and dissociation, and thus may inhibit platelet activation. The effect of colchicine on platelet activity is currently limited to in vitro studies or examination of platelet aggregation in clinical models [4-8]. The aim of the current study was to evaluate the effects of colchicine both in vitro and in vivo in healthy human subjects, with particular regard to homotypic versus platelet-leukocyte interactions.

\section{Methods}

\section{In vitro experiments}

Phlebotomy was performed in healthy subjects above the age of 21 years and not on any medications. After a $2 \mathrm{cc}$ discard, blood was collected in 3.2\% (0.109 mol/L) sodium citrate tubes (BD Vacutainer, $2.7 \mathrm{~mL}$ ). Good clinical practice protocols were followed to certify proper storage and daily and long-term quality control of reagents, instruments, and technique. Colchicine powder was obtained from Sigma Chemical Co. (St. Louis, MO) and diluted in PBS. Concentrations of colchicine used for the experiments described below were based on peak blood concentration $(6 \mathrm{ng} / \mathrm{mL}$ or $0.015 \mu \mathrm{M})$ after ingestion of $1.8 \mathrm{mg}$ PO load of colchicine over 1 hour [9].

Platelet aggregation was measured via light transmission aggregometry (LTA) using the AggRAM light transmission aggregometer (Helena Biosciences, Beaumont, TX). Briefly, citrate-anticoagulated blood was centrifuged at $200 \mathrm{~g}$ for 10 minutes to obtain platelet rich plasma (PRP) [10]. For these experiments, PRP was incubated with varying concentrations of colchicine $(0,0.015,0.15,1.5,15,150,1500,15000 \mu \mathrm{M})$ for 30 minutes at room temperature. LTA was immediately performed, and platelet aggregation was assessed in response to submaximal adenosine disphosphate (ADP) and epinephrine concentrations (1 $\mu \mathrm{M}$ and $0.4 \mu \mathrm{M}$, respectively) and recorded at maximum aggregation over a 10-minute evaluation.

Since an initial step of platelet activation is the adherence of platelets to leukocytes, the extent of monocyte- (MPA) and neutrophil-platelet aggregation (NPA) were investigated [11]. Citrate-anticoagulated blood was incubated with varying concentrations of colchicine $(0,0.015,0.15 \mu \mathrm{M})$ at room temperature, followed by $0.05 \mathrm{U}$ thrombin. The mixture was then stained with CD61-FITC (platelets) and CD14-APC (monocytes), and samples were evaluated immediately on an Accuri C6 flow cytometer (BD Biosciences, Franklin Lakes, NJ). MPA was assessed using CD14 to identify the monocyte population, and NPA was assessed using forward/side light scattering characteristics to identify the neutrophil population. Data are expressed as a percentage of all monocytes or neutrophils positive for adherent platelets. In these experiments, 2000 monocytes and 10,000 neutrophils per sample were analyzed [12-13]. 


\section{Healthy subjects study design}

We conducted a prospective study of healthy adults with no chronic medical problems or current prescription medication use. Exclusion criteria included the following: (1) history of cardiovascular disease; (2) history of myelodysplasia; (3) use of medications (prescription or over the counter) known to affect platelet function, including non-steroidal antiinflammatory drugs, anti-histamines, and selective serotonin reuptake inhibitors within 5 days of study procedures; (4) history of intolerance to colchicine; (5) symptoms of acute or chronic diarrhea, nausea or vomiting within 1 month of study procedures; (6) known anemia or hemoglobin $<10 \mathrm{mg} / \mathrm{dL}$; (7) platelet count $<100,000$ or $>450,000 \mathrm{~K} / \mu \mathrm{L}$; (8) creatinine clearance $<30 \mathrm{cc} /$ minute; (9) any known hemorrhagic diathesis; (10) current pregnancy; or (11) participation in a competing study. The study is registered at ClinicalTrials.gov (identifier: NCT02140372). The study was approved by the New York University School of Medicine Institutional Review Board and all subjects provided written informed consent.

Demographic information was self-reported. Height, weight, and abdominal circumference were measured by trained research nurses or assistants. Pertinent medical history was obtained from subject interview and questionnaire. Subjects fasted overnight and refrained from intensive exercise and tobacco use for 8 hours prior to study procedures. Phlebotomy was performed via standard venipuncture with a 19-guage needle. After a $2 \mathrm{cc}$ discard, blood was collected in 3.2\% (0.109 mol/L) sodium citrate tubes (BD Vacutainer, $2.7 \mathrm{~mL}$ ). Colchicine tablets $(0.6 \mathrm{mg})$ were obtained from the New York University Clinical Translational Science Institute investigational pharmacy. After obtaining a baseline blood sample, subjects were administered $1.2 \mathrm{mg}$ colchicine by mouth followed by $0.6 \mathrm{mg}$ colchicine by mouth 1 hour later. Subsequent blood samples were drawn 2 and 24 hours after completion of the $1.8 \mathrm{mg}$ colchicine loading dose.

\section{Measurements of platelet activation and adhesion}

For the evaluation of platelet activity via LTA, citrate-anticoagulated blood was centrifuged within 15 minutes of collection at 200g for 10 minutes to obtain PRP. LTA was performed 30 minutes later on the AggRAM light transmission aggregometer. Platelet aggregation was assessed in response to submaximal ADP and epinephrine concentrations and recorded at maximum aggregation over a 10-minute evaluation.

For the evaluation of platelet activity via extent of MPA and NPA, platelet surface PAC-1 expression, and platelet surface P-selectin expression, citrate-anticoagulated blood was processed within 15 minutes of collection. Whole blood was incubated with CD61-FITC or CD42b-APC (platelets), CD14-APC (monocytes), CD45-APC (leukocytes), PAC-1-FITC, and CD62-FITC (P-selectin) antibodies and evaluated on an Accuri C6 flow cytometer. MPA was assessed using CD14 to identify the monocyte population, and NPA was assessed using CD45 and forward/side light scattering characteristics to identify the neutrophil population. Monocytes and neutrophils with adherent platelets were identified by CD61/ CD42b positivity.

For the evaluation of platelet adhesion, platelets were isolated from citrate-anticoagulated blood and stained with $1 \mu \mathrm{M}$ DiO. Following a second isolation process, stained platelets 
were resuspended in Tyrodes buffer at a concentration of $1 \times 10^{5}$ platelets $/ \mu \mathrm{L}$. Coverslips $(18 \mathrm{~mm})$ were coated with $100 \mu \mathrm{g} / \mathrm{mL}$ collagen at $37^{\circ} \mathrm{C}$ for 1 hour, followed by addition of $0.1 \mathrm{U}$ thrombin prior to addition of $1 \mathrm{~mL}$ isolated stained platelets. Platelets were incubated at $37^{\circ} \mathrm{C}$ for 30 minutes, and the wells were then washed twice and analyzed using an Evos FL fluorescence microscope (Thermo Fisher Scientific Inc., Waltham, MA). Platelet adhesion was quantified using Image J software (U. S. National Institutes of Health, Bethesda, Maryland).

\section{Statistical analyses}

The study was designed as a pilot evaluation so no comparator group was studied. Continuous variables are presented as median [interquartile range] and categorical variables are presented as proportions. Markers of platelet activity before and at 2 and 24 hours after colchicine administration were compared with Wilcoxon signed-rank test for continuous variables (Statistical Package for the Social Sciences, IBM, Armonk, NY). Statistical significance was inferred at a two-sided p-value $<0.05$.

\section{Results \\ In vitro assessment}

In vitro addition of colchicine to PRP significantly decreased platelet-platelet aggregation only at concentrations $>1.5 \mu \mathrm{M}$ (Figure 1). Similar results were noted when colchicine was added to whole blood, and with colchicine incubation times ranging from 2 to 60 minutes (data not shown). In contrast, in vitro addition of colchicine to whole blood decreased MPA and NPA at a concentration of $0.015 \mu \mathrm{M}$ (Figure 2). Colchicine treated platelets demonstrated viability with characteristic forward/side light scattering pattern on flow cytometry at all the examined concentrations of colchicine.

\section{In vivo colchicine administration-prospective healthy subject cohort}

Baseline characteristics of the study cohort $(\mathrm{n}=10)$ are shown in Table 1 . The $1.8 \mathrm{mg}$ oral load of colchicine had no significant effect on platelet aggregation in response to $1 \mu \mathrm{M}$ ADP and $0.4 \mu \mathrm{M}$ epinephrine (Figure 3). In contrast, colchicine administration significantly decreased the extent of MPA and NPA at 2 hours but not at 24 hours (Figure 4). Platelet surface expression of PAC-1 and P-selectin also significantly decreased 2 hours after the 1.8 $\mathrm{mg}$ load of colchicine, an effect that was sustained for PAC-1 but not P-selectin expression at 24 hours (Figure 5).

In subjects with available platelet/collagen adhesion data $(\mathrm{n}=8)$, we observed a numerical, but not statistically significant, decrease in the proportion of platelets adherent to collagen after the administration of colchicine (Figure 6). However, when the one subject with increasing adherence over time was removed, the proportion of platelets adherent to collagen was significantly lower by 24 hours after the administration of colchicine $(\mathrm{p}=0.018)$. 


\section{Discussion}

Recent data suggest that colchicine may be effective in decreasing cardiovascular events. While colchicine is used clinically as an anti-inflammatory drug, in vitro studies suggest that platelet microtubules may be an additional target of this drug. This study demonstrated that the current standard of care $1.8 \mathrm{mg}$ loading dose of oral colchicine decreases platelet activity as it relates to platelet activation and the platelet-leukocyte interface, but may not directly impact on platelet-platelet interactions.

Our in vitro data suggest that addition of colchicine for 30 minutes decreases platelet aggregation only at supratherapeutic concentrations $(>0.015 \mu \mathrm{M})$. This finding is consistent with a study by Karpatkin et al that demonstrated a decrease in platelet aggregation in response to ADP, epinephrine, and collagen when PRP was pre-incubated for 2 minutes with $250 \mu \mathrm{M}$ to $2000 \mu \mathrm{M}$ colchicine but not at lower concentrations [4]. Moreover, Bouaziz et al observed a decrease in microtubular content and tubulin polymerization when thrombinstimulated platelets were pre-incubated for a longer time period (120 minutes) with $10 \mu \mathrm{M}$ to $1000 \mu \mathrm{M}$ colchicine, but demonstrated a modest $20 \%$ decrease in platelet aggregation only when these platelets were treated with $100 \mu \mathrm{M}$ to $1000 \mu \mathrm{M}$ colchicine [14]. Although the current study used a 30 minute colchicine incubation time, we also observed no difference between colchicine incubation times of 2 minutes and 60 minutes. Since we did not use thrombin as an agonist, we cannot comment on the aggregation response to thrombin in the current study.

Mechanistic in vitro studies suggest that therapeutic concentrations of colchicine bind to platelet tubulin and modulate microtubular associated protease, which in turn either prevents phosphoprotein synthesis or degrades existing phospoproteins required for microtubular polymerization $[4,15]$. Based on our study, this putative mechanism of action may not translate into functional effects on platelet aggregation in the setting of therapeutic oral colchicine administration. One pilot study did evaluate the effect of oral colchicine $1 \mathrm{mg}$ per day in 80 patients with acute coronary syndrome or acute ischemic stroke on a background of aspirin therapy, $85 \%$ of which were on dual anti-platelet therapy with clopidogrel [16]. In a subgroup of 49 patients with platelet aggregation measured, there was no noted difference in platelet aggregation in response to ADP, arachadonic acid, or collagen [16]. Likewise, another study demonstrated no difference in mean platelet volume, a marker of platelet activity based on size, between patients with familial mediterranean fever on colchicine and age- and sex-matched healthy subjects [17-18]. Overall, these data suggest that colchicine may not inhibit platelet homotypic aggregation within the pharmacologically achievable range in humans.

Colchicine does, however, exert anti-inflammatory effects by decreasing neutrophil surface expression of L-selectin; suppressing neutrophil tyrosine phosphorylation and subsequent granule enzyme release; decreasing IL-1 $\beta$ production by the NLRP3 inflammasome; and decreasing qualitative expression of E-selectin on endothelial cells [19-21]. In the current study, we observed decreases in both MPA and NPA after in vitro addition of therapeutic concentrations of colchicine, as well as after oral administration of the standard $1.8 \mathrm{mg}$ colchicine load. MPA and leukocyte platelet aggregates (LPA) are robust markers of platelet 
adhesion, and levels are elevated in patients with coronary artery disease, an inflammatory process, as well as associated with models of myocardial reperfusion injury [22-24]. Likewise, PAC-1, a mediator of platelet-to-platelet adhesion, and P-selectin, a mediator of platelet-to-leukocyte adhesion, both decreased after oral administration of colchicine.

The decrease in adhesion to collagen observed after oral administration of colchicine was apparent at 2- and 24- hours. The lack of statistical significance was likely due to the limited statistical power of our small sample size. In fact, after excluding a single outlier in our dataset, the adhesion was significantly lower following colchicine. One prior study evaluated the effects of a 60 minute incubation of platelet suspensions with a supratherapeutic concentration of colchicine $(10,000 \mu \mathrm{M})$ and noted partial inhibition of platelet adhesion to glass slides [25]. Those platelets also demonstrated partial membrane extension with short filopodia.

Our study has a number of limitations. As a mechanistic pilot study evaluating the effects of colchicine on several markers of platelet activity in 10 healthy subjects, the effect of colchicine on platelet aggregation in a larger population, as well as the clinical ramifications of the results presented herein, remain uncertain. Furthermore, representative markers of platelet activity were evaluated, but only a limited number of agonist concentrations were tested in the setting of LTA. Finally, the effects of colchicine on a background of antiplatelet therapy were not evaluated. Nonetheless, we note that prior studies by Nidorf et al suggest that colchicine may have cardioprotective effects even among patients concurrently taking aspirin. We believe the data presented herein provide biological plausibility for larger studies examining the effects of colchicine use on platelet activity in patients with coronary artery disease on aspirin monotherapy, as well as those on dual anti-platelet therapy with aspirin and a P2Y12 inhibitor. Taken together, our data suggest the possibility that in vivo, colchicine may have a beneficial cardiovascular effect by targeting the plateletinflammatory axis. Whether targeting this pathway in vivo can offer protection from atherothrombosis without increasing the bleeding risk observed with traditional plateletdirected therapies remains unknown and deserving of future investigation.

\section{Acknowledgments}

This study was supported in part by the NYU CTSA grant UL1TR000038 from the National Center for Advancing Translational Sciences (NIH). Dr. Shah was partially funded by the American Heart Association Clinical Research Program (13CRP14520000) and the New York State Department of Health Empire Clinical Research Investigator Program. Dr. Berger was partially funded by the National Heart and Lung Blood Institute of the National Institutes of Health (RO1 HL1 14978), American Heart Association Clinical Research Program (13CRP14410042) and Doris Duke Charitable Foundation (2010055).

\section{References}

1. Slobodnick A, Shah B, Pillinger MH, Krasnokutsky S. Colchicine: Old and New. Am J Med. 2014 Dec 30. Epub ahead of print.

2. Crittenden DB, Lehmann RA, Schneck L, Keenan RT, Shah B, Greenberg JD, Cronstein BN, Sedlis SP, Pillinger MH. Colchicine use is associated with decreased prevalence of myocardial infarction in patients with gout. J Rheumatol. 2012; 39:1458-1464. [PubMed: 22660810]

3. Nidorf SM, Eikelboom JW, Budgeon CA, Thompson PL. Low-Dose Colchicine for Secondary Prevention of Cardiovascular Disease. J Am Coll Cardiol. 2013; 61:404-10. [PubMed: 23265346] 
4. Menche D, Israel A, Karpatkin S. Platelets and microtubules. Effect of colchicine and D2O on platelet aggregation and release induced by calcium ionophore A23187. J Clin Invest. 1980; 66:284-91. [PubMed: 6772671]

5. Behnke O. Further studies on microtubules. A marginal bundle in human and rat thrombocytes. J Ultrastruct Res. 1965; 13:469-77. [PubMed: 5848841]

6. White JG. Fine structural alterations induced in platelets by adenosine diphosphate. Blood. 1968; 31:604-22. [PubMed: 5646304]

7. White JG. Effects of colchicine and Vinca alkaloids on human platelets. I. Influence on platelet microtubules and contractile function. Am J Pathol. 1968; 53:281-91. [PubMed: 5667580]

8. White JG. Effects of colchicine and vinca alkaloids on human platelets. 3. Influence on primary internal contraction and secondary aggregation. Am J Pathol. 1969; 54:467-78. [PubMed: 5774266]

9. Terkeltaub RA, Furst DE, Bennett K, Kook KA, Crockett RS, Davis MW. High versus low dosing of oral colchicine for early acute gout flare. Arthritis and Rheumatism. 2010; 62:1060-8. [PubMed: 20131255]

10. Merolla M, Nardi MA, Berger JS. Centrifugation speed affects light transmission aggregometry. Int J Lab Hematol. 2012; 34:81-85. [PubMed: 21794095]

11. Furman MI, Benoit SE, Barnard MR, Valeri CR, Borbone ML, Becker RC, Hechtman HB, Michelson AD. Increased platelet reactivity and circulating monocyte-platelet aggregates in patients with stable coronary artery disease. J Am Coll Cardiol. 1998; 31:352-358. [PubMed: 9462579]

12. Shah B, Sedlis SP, Mai X, Amoroso NS, Guo Y, Lorin JD, Berger JS. Comparison of platelet activity measurements using arterial and venous blood sampling. J Thromb Haemost. 2013; 11:1922-1924. [PubMed: 23927560]

13. Shah B, Valdes V, Nardi MA, Hu L, Schrem E, Berger JS. Mean platelet volume reproducibility and association with platelet activity and anti-platelet therapy. Platelets. 2014; 25:188-192. [PubMed: 23786366]

14. Bouaziz A, Amor NB, Woodard GE, Zibidi H, Lopez JJ, Bartegi A, Salido GM, Rosado JA. Tyrosine phosphorylation/dephosphorylation balance is involved in thrombin-evoked microtubular reorganization in human platelets. Thromb Haemost. 2007; 98:375-384. [PubMed: 17721620]

15. Wittels E, Israel A, Karpatkin S. Evidence for colchicine-dependent protease activity in human platelets. Thromb Res. 1981; 24:215-221. [PubMed: 7038973]

16. Raju NC, Yi Q, Nidorf M, Fagel ND, Hiralal R, Eikelboom JW. Effect of colchicine compared with placebo on high sensitivity C-reactive protein in patients with acute coronary syndrome or acute stroke: a pilot randomized controlled trial. J Thromb Thrombolysis. 2012; 33:88-94. [PubMed: 21918905]

17. Shah B, Valdes V, Nardi MA, Hu L, Schrem E, Berger JS. Mean platelet volume reproducibility and association with platelet activity and anti-platelet therapy. Platelets. 2014; 25:188-192. [PubMed: 23786366]

18. Abanonu GB, Daskin A, Akdogan MF, Uyar S, Demirunc R. Mean platelet volume and Bthromboglobulin levels in familial Mediterranean fever: Effect of colchicine use? Eur J Intern Med. 2012; 23:661-664. [PubMed: 22939812]

19. Cronstein BN, Molad Y, Reibman J, Balakhane E, Levin RI, Weissmann G. Colchicine alters the quantitative and qualitative display of selectins on endothelial cells and neutrophils. J Clin Invest. 1995; 96:994-1002. [PubMed: 7543498]

20. Roberge CJ, Gaudry M, de Médicis R, Lussier A, Poubelle PE, Naccache PH. Crystal-induced neutrophil activation. IV. Specific inhibition of tyrosine phosphorylation by colchicine. J Clin Invest. 1993; 92:1722-1729. [PubMed: 7691884]

21. Martinon F, Pétrilli V, Mayor A, Tardivel A, Tschopp J. Gout-associated uric acid crystals activate the NALP3 inflammasome. Nature. 2006; 440:237-241. [PubMed: 16407889]

22. Furman MI, Barnard MR, Krueger LA, Fox ML, Shilale EA, Lessard DM, Marchese P, Frelinger AL 3rd, Goldberg RJ, Michelson AD. Circulating monocyte-platelet aggregates are an early marker of acute myocardial infarction. J Am Coll Cardiol. 2001; 38:1002-1006. [PubMed: 11583872] 
23. Mickelson JK, Lakkis NM, Villarreal-Levy G, Hughes BJ, Smith CW. Leukocyte activation with platelet adhesion after coronary angioplasty: a mechanism for recurrent disease? J Am Coll Cardiol. 1996; 28:345-353. [PubMed: 8800108]

24. Hayward R, Campbell B, Shin YK, Scalia R, Lefer AM. Recombinant soluble p-selectin glycoprotein ligand-1 protects against myocardial ischemic reperfusion injury in cats. Cardiovasc Res. 1999; 41:65-76. [PubMed: 10325954]

25. Cercedo D, Stock R, Gonzalez S, Reyes E, Mondragon R. Modification of actin, myosin and tubulin distribution during cytoplasmic granule movements associated with platelet adhesion. Haematologica. 2002; 87:1165-1176. [PubMed: 12414346] 


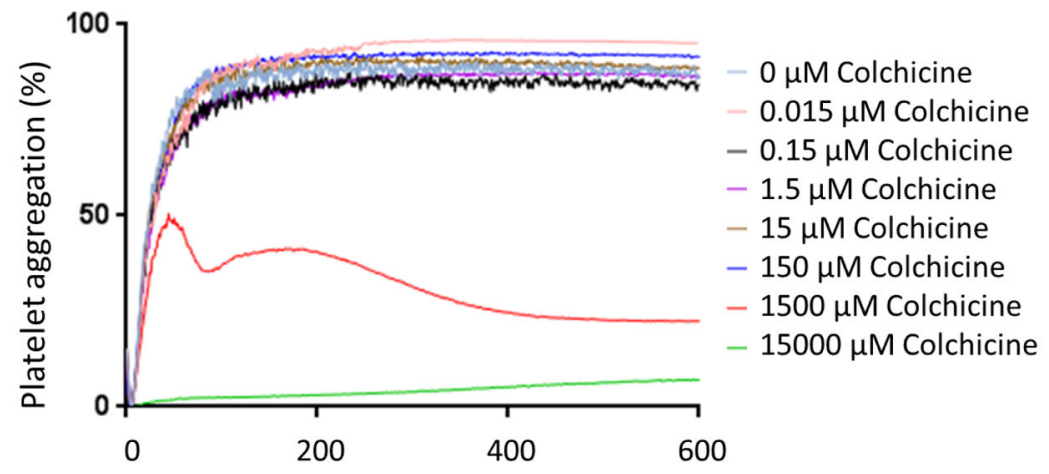

Time, seconds

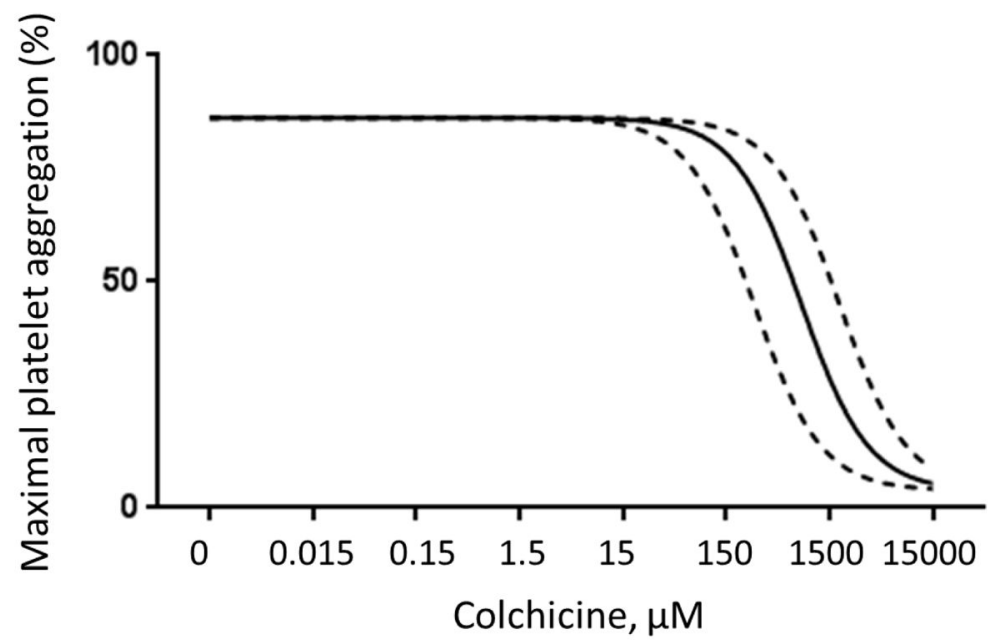

$\begin{array}{ccccccccc}\text { Median } & 89.2 & 91.8 & 90.5 & 90.4 & 87.7 & 83.7 & 26.6^{*} & 4.5^{*} \\ \text { 25th Percentile } & 77.0 & 83.3 & 86.6 & 57.2 & 66.1 & 60.9 & 17.0 & 3.9 \\ \text { 75\% Percentile } & 89.9 & 93.9 & 93.4 & 91.5 & 91.3 & 90.5 & 50.7 & 6.5\end{array}$

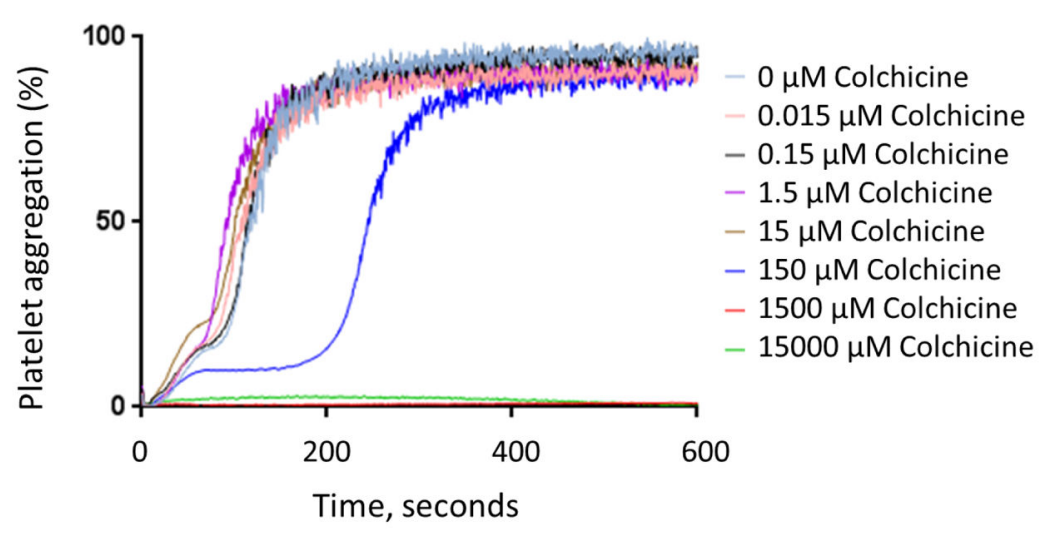

Inflammation. Author manuscript; available in PMC 2017 February 01. 


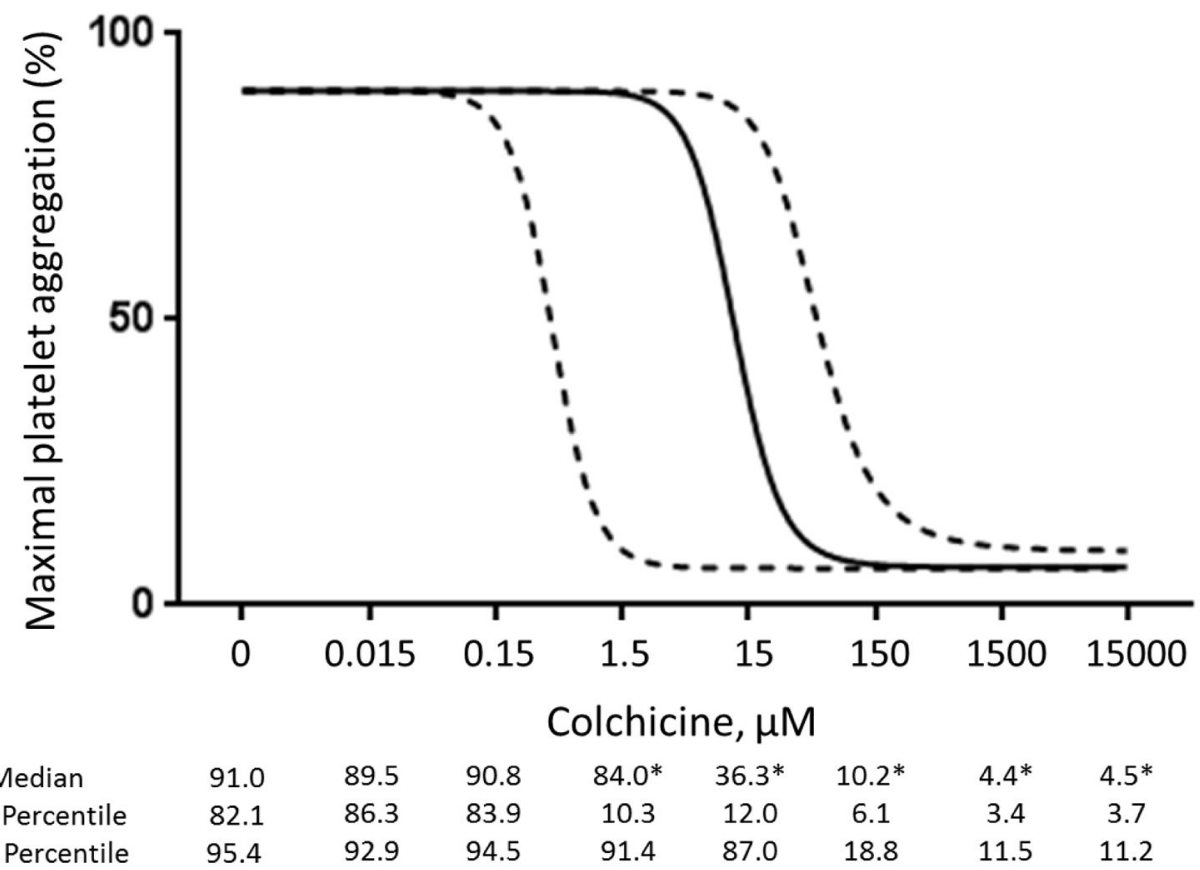

Figure 1. Effect of in vitro addition of colchicine to platelet rich plasma on platelet aggregation Platelet rich plasma was incubated with varying concentrations of colchicine for $30 \mathrm{~min}$. Maximal platelet aggregation was assessed in response to $2 \mu \mathrm{M}$ adenosine disphosphate (Representative response curves shown in Panel A, Median and interquartile range of 5 to 6 experiments shown in Panel B) and $1 \mu \mathrm{M}$ epinephrine (Representative response curves shown in Panel C, Median and interquartile range of 7 experiments shown in Panel D) over a 10 -minute evaluation. $*$ denotes $\mathrm{p}<0.05$ compared to no colchicine control. 

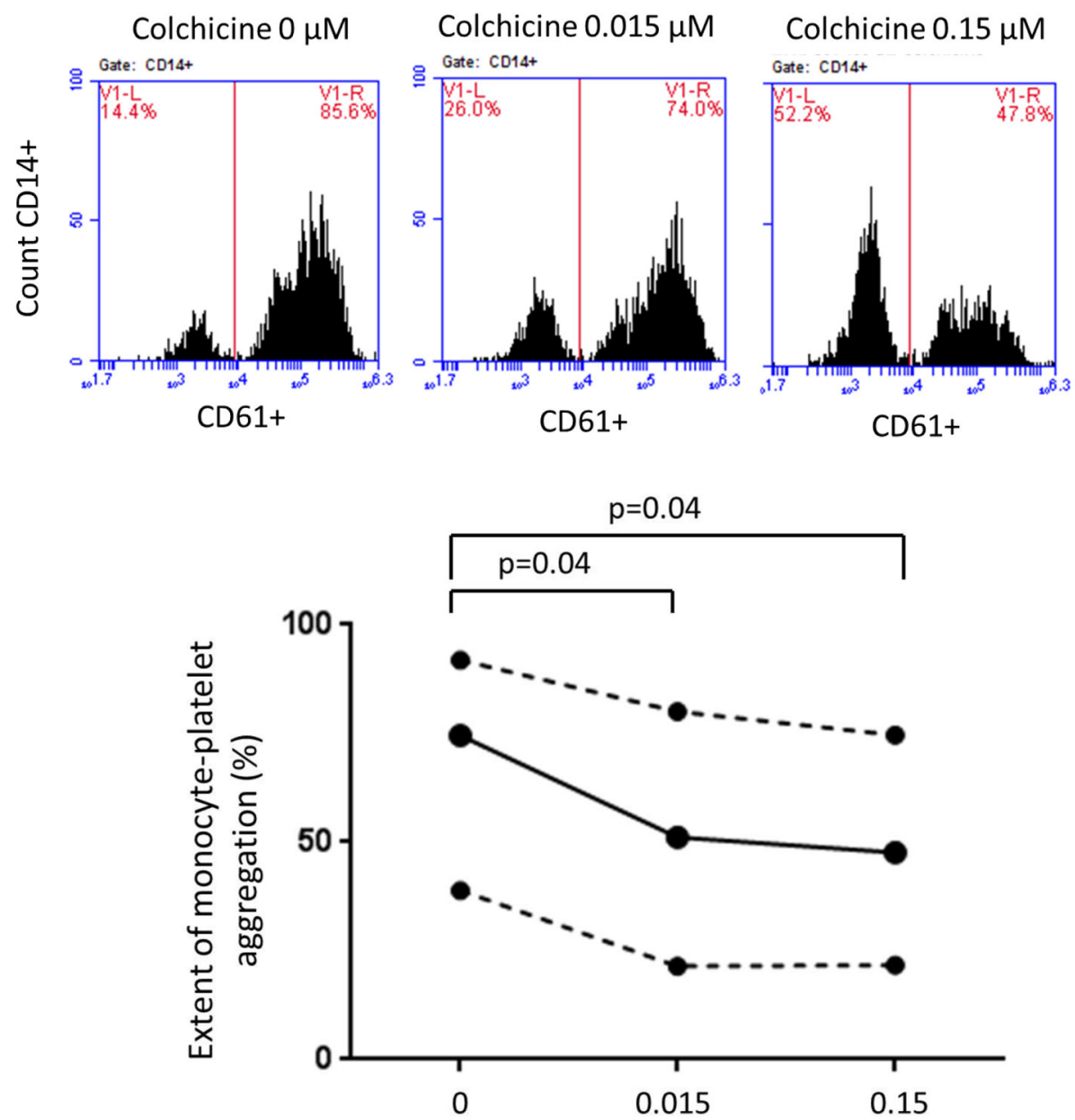

Colchicine, $\mu \mathrm{M}$

Median

74.5

51.1

47.6

[Interquartile range] [38.9-91.9]

[21.5-80.0]

[21.7-74.7]

\section{Colchicine $0 \mu \mathrm{M}$}

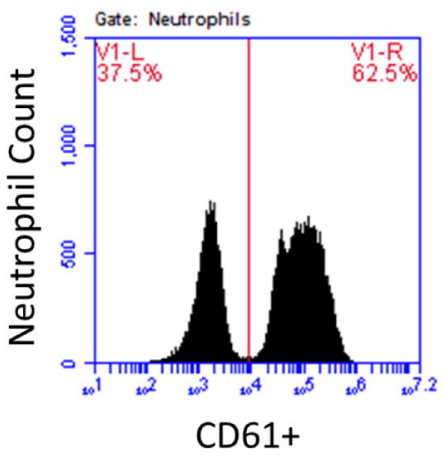

Colchicine $0.015 \mu \mathrm{M}$

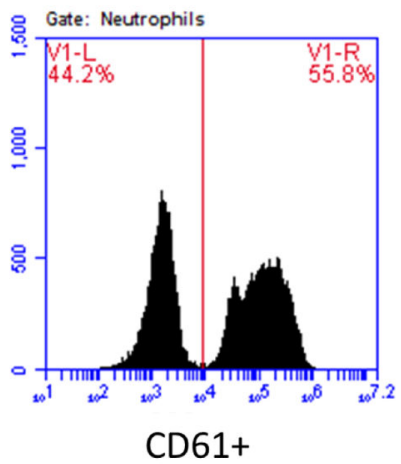

Colchicine $0.15 \mu \mathrm{M}$

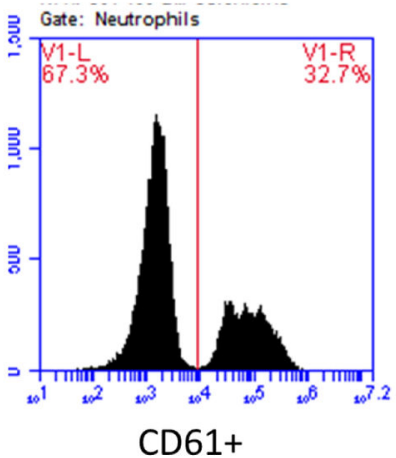

Inflammation. Author manuscript; available in PMC 2017 February 01. 

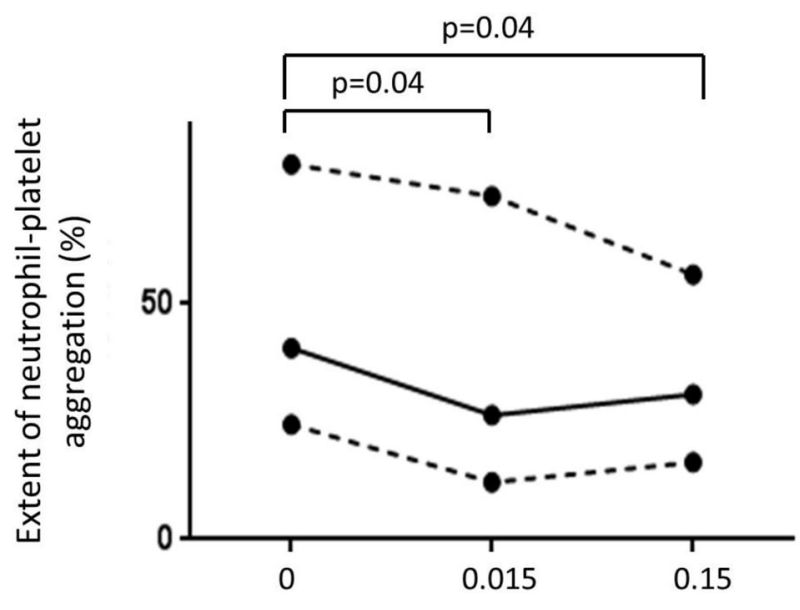

Colchicine, $\mu \mathrm{M}$

$\begin{array}{llll}\text { Median } & 40.6 & 26.4 & 30.8\end{array}$

[Interquartile range] [24.4-79.6] [12.2-72.9] [16.4-56.2]

Figure 2. Effect of in vitro addition of colchicine to whole blood on monocyte- and neutrophilplatelet aggregates

Whole blood was incubated with varying concentrations of colchicine for 5 minutes. Extent of monocyte-platelet aggregation (Representative gating images shown in Panel A, Median and interquartile range of 5 experiments shown in Panel B) and neutrophil-platelet aggregation (Representative gating images shown in Panel C, Median and interquartile range of 5 experiments shown in Panel D) were assessed in response to $0.05 \mathrm{U}$ thrombin. 


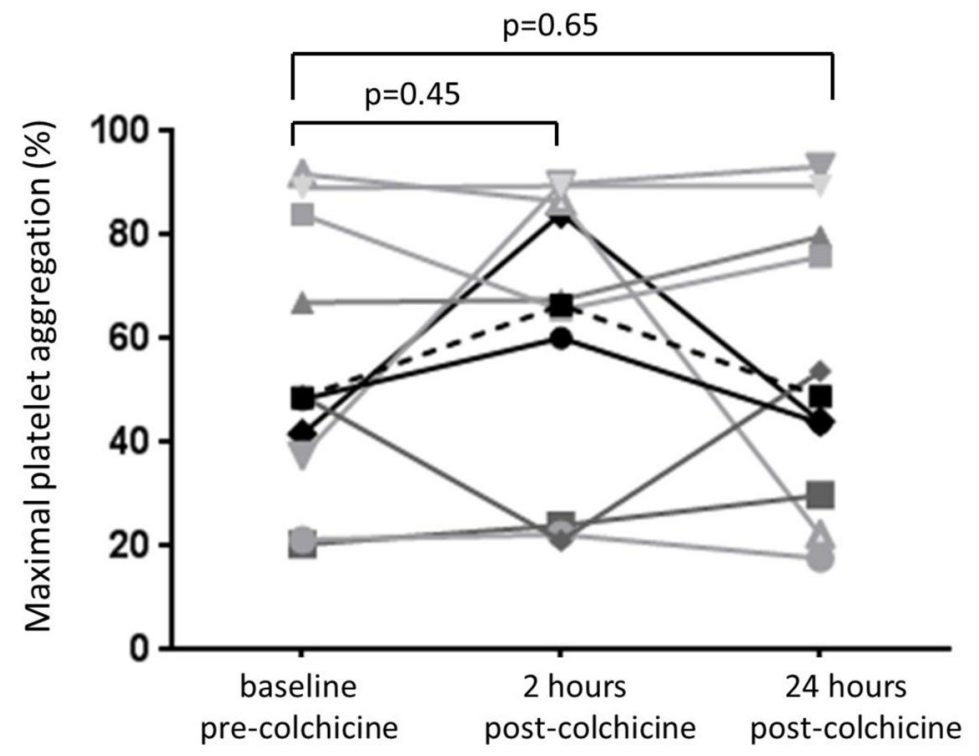

$$
\begin{array}{cccc}
\text { Median } & 48.7 & 66.6 & 49.0 \\
\text { [Interquartile range] } & {[33.5-85.3]} & {[23.6-87.3]} & {[28.0-82.2]}
\end{array}
$$

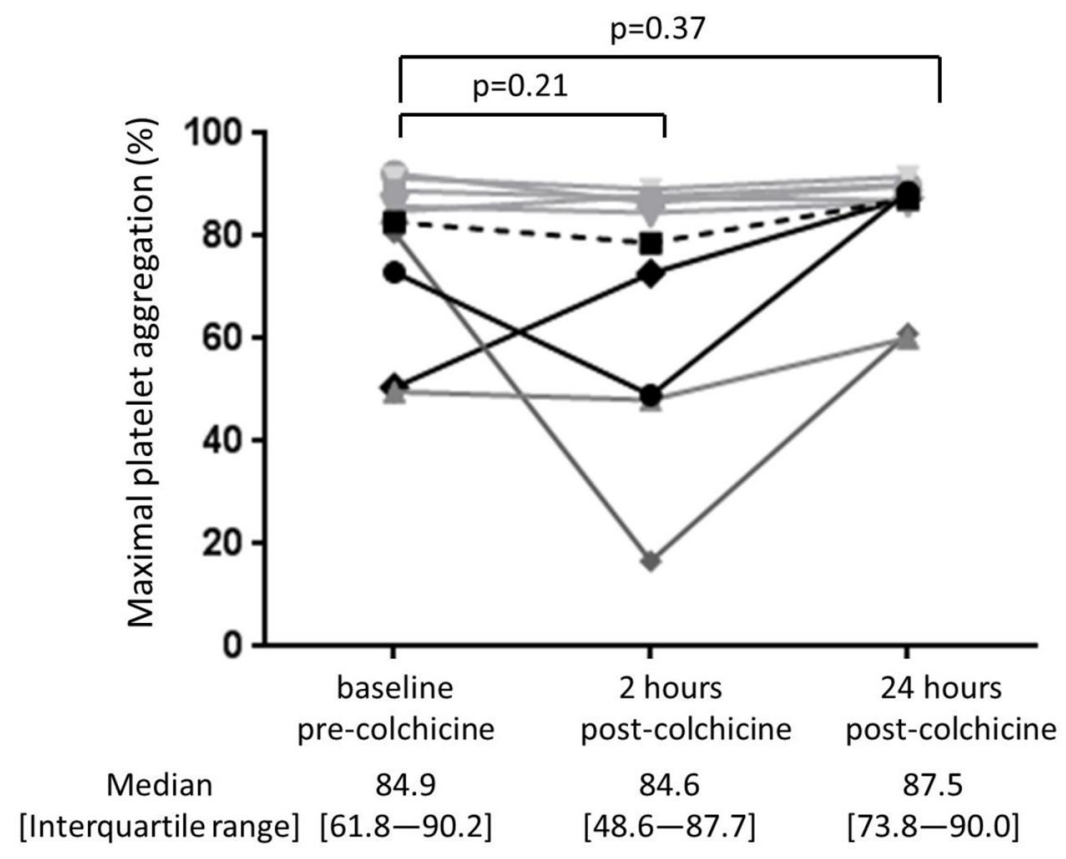

Figure 3. Effect of a $1.8 \mathrm{mg}$ load of colchicine on platelet aggregation

Maximal platelet aggregation was assessed before and 2 and 24 hours after completion of the $1.8 \mathrm{mg}$ load of colchicine in response to $1 \mu \mathrm{M}$ adenosine disphosphate (Panel A) and 0.4 $\mu \mathrm{M}$ epinephrine (Panel B) over a 10-minute evaluation in healthy subjects $(\mathrm{n}=10)$. Medians shown via dashed line. 

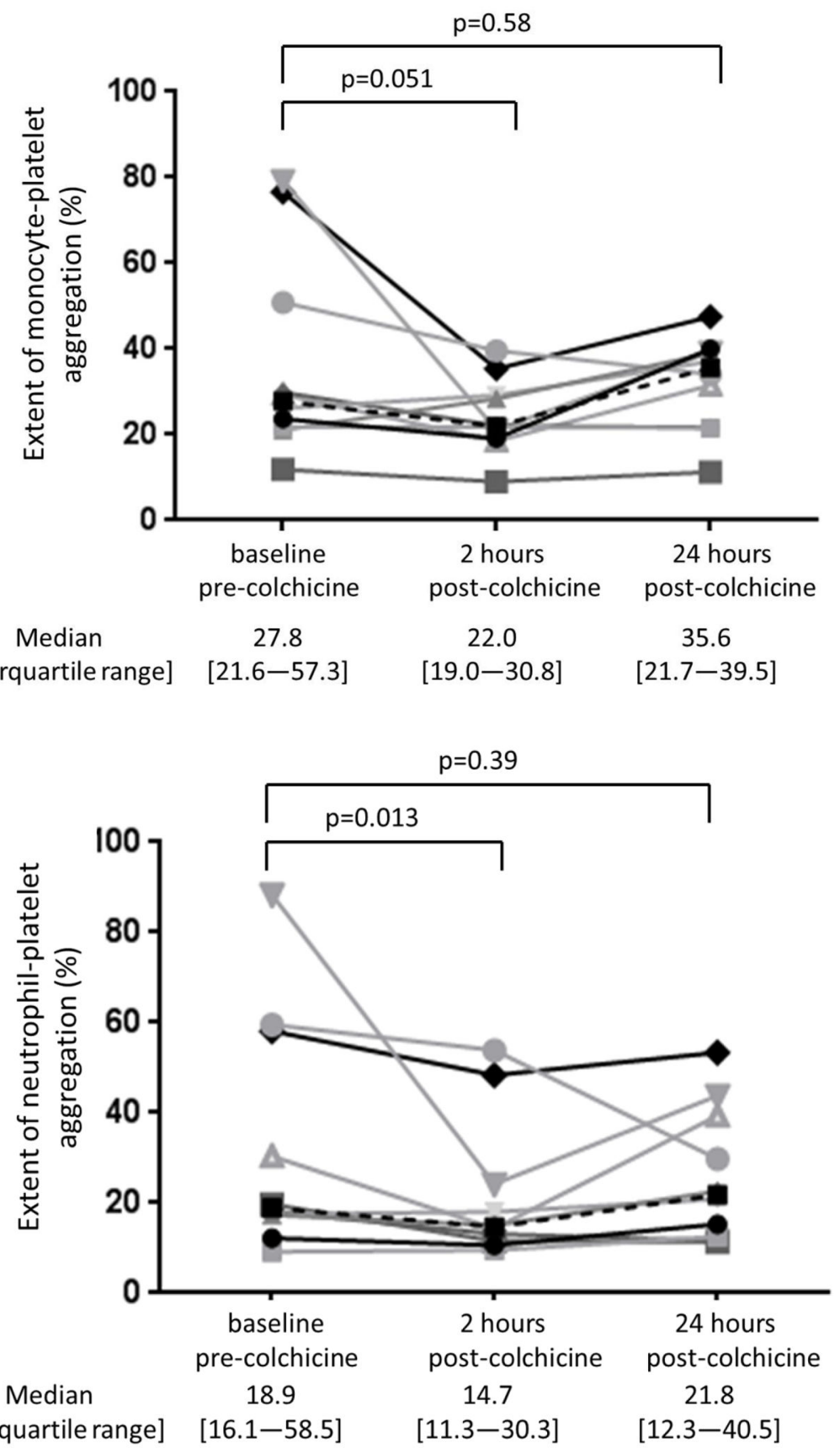

Figure 4. Effect of a $1.8 \mathrm{mg}$ load of colchicine on extent of monocyte- and neutrophil-platelet aggregate

Platelet surface expression of PAC-1 in response to $0.4 \mu \mathrm{M}$ epinephrine (Panel A) and Pselectin in response to $0.025 \mathrm{U}$ thrombin (Panel B) were assessed before and 2 and 24 hours after completion of the $1.8 \mathrm{mg}$ load of colchicine in healthy subjects $(n=10)$. Medians shown via dashed line. 

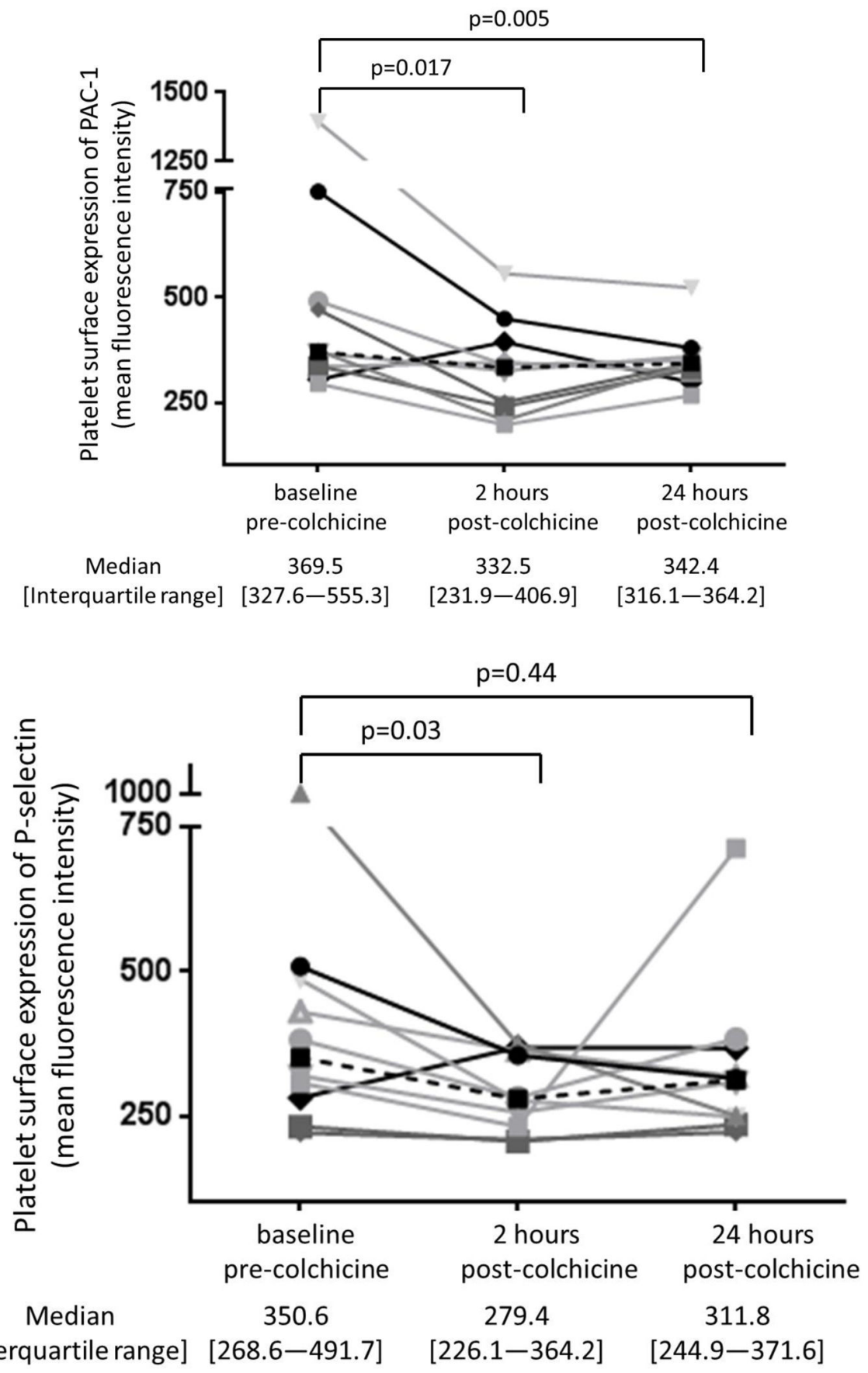

Figure 5. Effect of a $1.8 \mathrm{mg}$ load of colchicine on platelet surface expression of PAC-1 and Pselectin

Extent of monocyte-platelet aggregate (Panel A) and neutrophil-platelet aggregate (Panel B) were assessed before and 2 and 24 hours after completion of the $1.8 \mathrm{mg}$ load of colchicine in healthy subjects $(n=10)$. Medians shown via dashed line. 
Baseline pre-colchicine
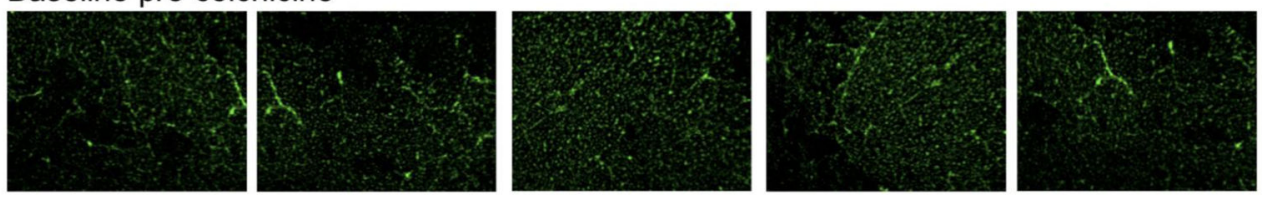

2 hours post-colchicine
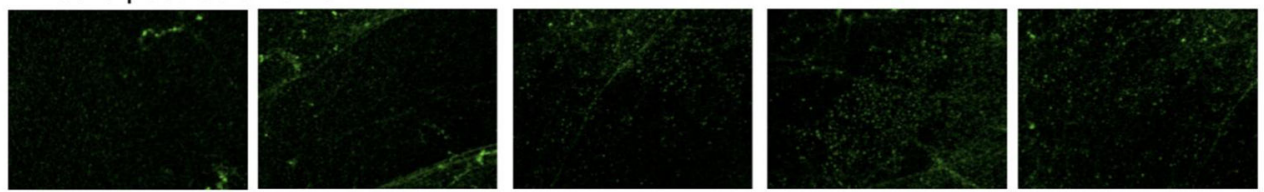

24 hours post-colchicine
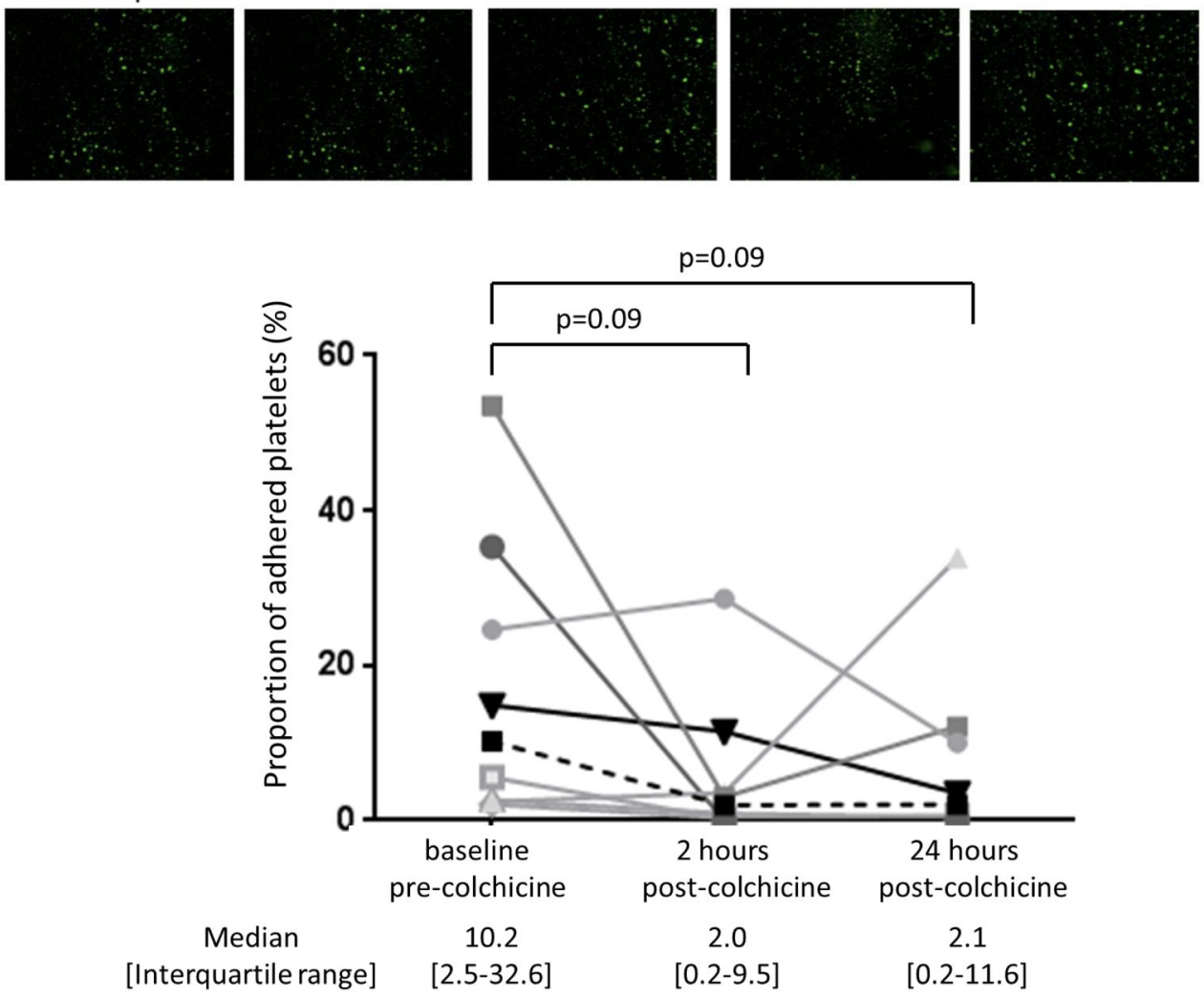

Figure 6. Effect of a $1.8 \mathrm{mg}$ load of colchicine on proportion of adhered platelets Proportion of platelets adhered to collagen stimulated by $0.1 \mathrm{U}$ thrombin was assessed before and 2 and 24 hours after completion of the $1.8 \mathrm{mg}$ load of colchicine in healthy subjects $(n=8)$. Representative adhesion assays shown in panel A. Individual data shown in panel B. Medians shown via dashed line.

Inflammation. Author manuscript; available in PMC 2017 February 01. 


\section{Table 1}

\section{Baseline characteristics}

\begin{tabular}{lc}
\hline & Healthy Subjects $(\mathbf{n}=\mathbf{1 0})$ \\
\hline Age, years & $26[24-41]$ \\
Male sex & 6 \\
Race & 7 \\
$\quad$ White & 2 \\
Black & 1 \\
Asian & \\
Ethnicity & 3 \\
Hispanic & $24.6[22.5-27.9]$ \\
Body mass index, kg/m² & $81[76-88]$ \\
Abdominal circumference, cm & 3 \\
History of tobacco use & 1 \\
Family history of cardiovascular disease & \\
Laboratory data & $13.2[12.4-14.2]$ \\
Hemoglobin level, g/dL & $209[189-220]$ \\
Platelet count, K/uL & $5.4[4.1-6.4]$ \\
White blood cell count, K/uL & $1.8[1.4-3.1]$ \\
Neutrophil/lymphocyte ratio &
\end{tabular}

- Continuous variables presented as median [interquartile range]

-Categorical variables presented as $n$ 